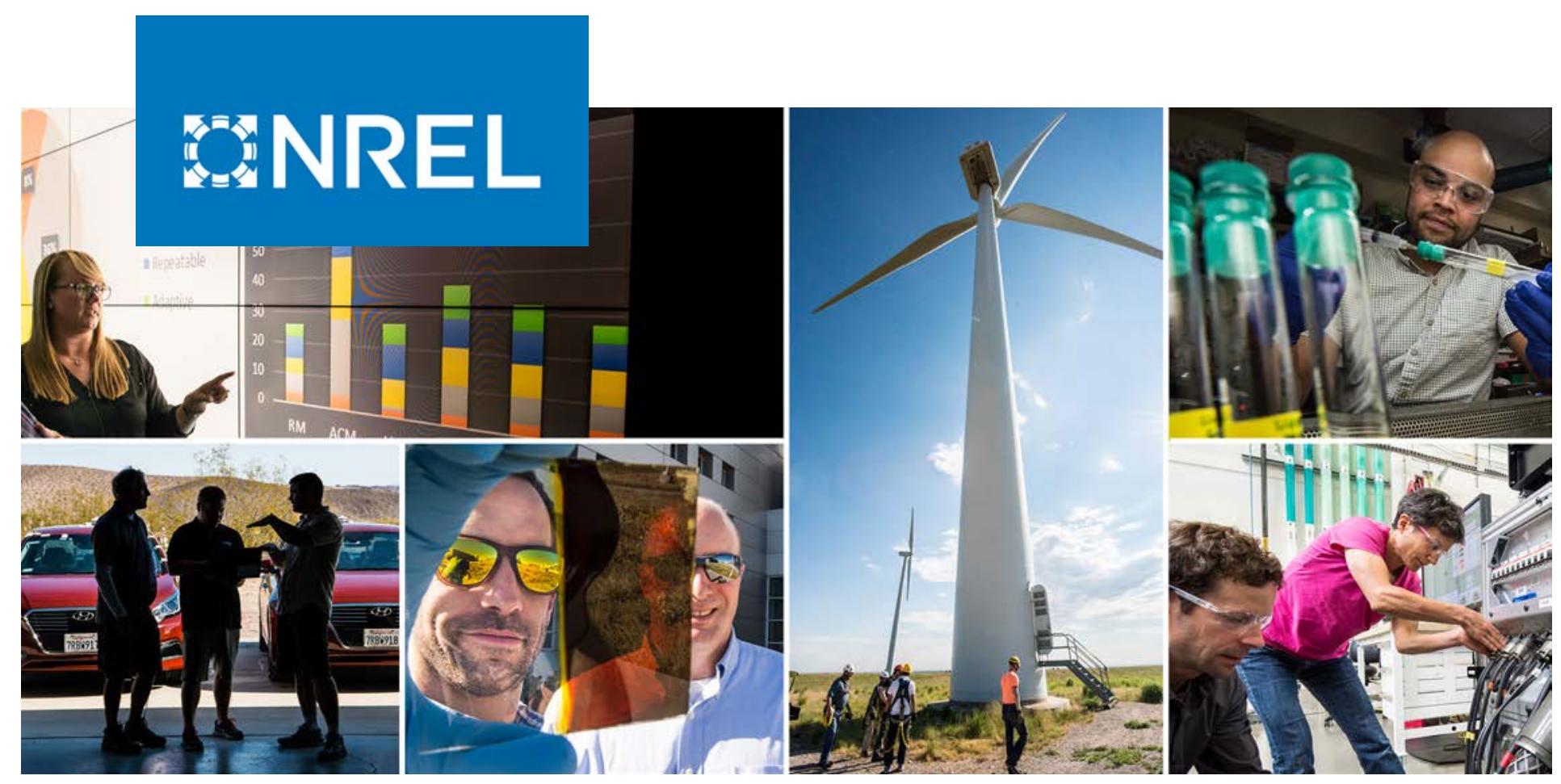

\title{
Implementing Inertial Control for PMSG-WTG in Region 2 Using Virtual Synchronous Generator with Multiple Virtual Rotating Masses
}

\section{Preprint}

Weihang Yan, ${ }^{1}$ Wei Gao, ${ }^{1}$ Wenzhong Gao, ${ }^{1}$ and Vahan Gevorgian²

1 University of Denver

${ }^{2}$ National Renewable Energy Laboratory

Presented at the IEEE Power and Energy Society General Meeting (IEEE PES GM) Atlanta, Georgia

August 4-8, 2019

NREL is a national laboratory of the U.S. Department of Energy Office of Energy Efficiency \& Renewable Energy

Operated by the Alliance for Sustainable Energy, LLC

This report is available at no cost from the National Renewable Energy Laboratory (NREL) at www.nrel.gov/publications.
Conference Paper

NREL/CP-5D00-73872

February 2020 


\title{
GNREL
}

\section{Implementing Inertial Control for PMSG-WTG in Region 2 Using Virtual Synchronous Generator with Multiple Virtual Rotating Masses}

\section{Preprint}

\author{
Weihang Yan, ${ }^{1}$ Wei Gao, ${ }^{1}$ Wenzhong Gao, ${ }^{1}$ \\ and Vahan Gevorgian ${ }^{2}$ \\ ${ }^{1}$ University of Denver \\ ${ }^{2}$ National Renewable Energy Laboratory
}

\section{Suggested Citation}

Yan, Weihang, Wei Gao, Wenzhong Gao, and Vahan Gevorgian. 2020. Implementing Inertial Control for PMSG-WTG in Region 2 Using Virtual Synchronous Generator with Multiple Virtual Rotating Masses: Preprint. Golden, CO: National Renewable Energy Laboratory. NREL/CP-5D00-73872. https://www.nrel.gov/docs/fy20osti/73872.pdf.

(c) 2020 IEEE. Personal use of this material is permitted. Permission from IEEE must be obtained for all other uses, in any current or future media, including reprinting/republishing this material for advertising or promotional purposes, creating new collective works, for resale or redistribution to servers or lists, or reuse of any copyrighted component of this work in other works.

NREL is a national laboratory of the U.S. Department of Energy Office of Energy Efficiency \& Renewable Energy Operated by the Alliance for Sustainable Energy, LLC

This report is available at no cost from the National Renewable Energy Laboratory (NREL) at www.nrel.gov/publications.

Contract No. DE-AC36-08GO28308
Conference Paper

NREL/CP-5D00-73872

February 2020

National Renewable Energy Laboratory 15013 Denver West Parkway Golden, CO 80401

303-275-3000 • www.nrel.gov 


\section{NOTICE}

This work is supported by US National Science Foundation Grant 1711951 and 1429093 . This work was authored in part by the National Renewable Energy Laboratory, operated by Alliance for Sustainable Energy, LLC, for the U.S. Department of Energy (DOE) under Contract No. DE-AC36-08G028308. Funding provided U.S. Department of Energy Office of Energy Efficiency and Renewable Energy Wind Energy Technologies Office (WETO). The views expressed herein do not necessarily represent the views of the DOE or the U.S. Government. The U.S. Government retains and the publisher, by accepting the article for publication, acknowledges that the U.S. Government retains a nonexclusive, paid-up, irrevocable, worldwide license to publish or reproduce the published form of this work, or allow others to do so, for U.S. Government purposes.

The authors thank Charlton Clark and Jian Fu of DOE WETO for their continuous support this work.

This report is available at no cost from the National Renewable Energy Laboratory (NREL) at www.nrel.gov/publications.

U.S. Department of Energy (DOE) reports produced after 1991 and a growing number of pre-1991 documents are available free via www.OSTI.gov.

Cover Photos by Dennis Schroeder: (clockwise, left to right) NREL 51934, NREL 45897, NREL 42160, NREL 45891, NREL 48097, NREL 46526.

NREL prints on paper that contains recycled content. 


\section{Implementing Inertial Control for PMSG-WTG in Region 2 using Virtual Synchronous Generator with Multiple Virtual Rotating Masses}

\author{
Weihang Yan, Wei Gao, Wenzhong Gao \\ Dept. Electrical and Computer Engineering \\ University of Denver \\ Denver, CO.
}

\author{
Vahan Gevorgian \\ Power System Engineering Center \\ National Renewable Energy Laboratory \\ Golden, $\mathrm{CO}$.
}

\begin{abstract}
With the increasing integration of renewable energy, the problems associated with deteriorating grid frequency profile and potential power system instability have become more significant. In this paper, the inertial control algorithm using Virtual Synchronous Generator (VSG) is implemented on type-4 Permanent Magnet Synchronous Generator (PMSG) - wind turbine generator (WTG). The overall nonlinear dynamic model and its small-signal linearization of PMSG-WTG using VSG is established and comprehensively analyzed. Inevitably, the direct application of VSG introduces large inertia which causes conflict between the fast-varying of available wind power and inverter control with slow dynamics, particularly in region 2 of wind turbine. Aiming to address such issue, VSG with multiple virtual rotating masses is proposed in order to improve the active power tracking performance as well as to boost inertial control of a VSG. The inertial responses are verified in a modified 10MVA IEEE 14 bus microgrid system. The assessment of the simulation results demonstrates the applicability of VSG on renewable energy generation units.
\end{abstract}

Index Terms-- inertial control, Virtual Synchronous Generator, wind power, multiple virtual rotating masses.

\section{INTRODUCTION}

Wind turbines inherently store large amount of kinetic energy within their turbine blades and rotor shafts. Such beneficial feature in fact makes a WTG capable of providing inertia response without the necessity of using energy storage system (ESS). Generally, most available literatures consider inertial control as an add-on function for WTG. Existing WTG inertial control algorithms for type-4 wind energy conversion system (WECS) can be summarized into two categories: frequency-based inertial control (FBIC) [1], [2], [6], [7] and stepwise inertial control (SIC) [3]-[7]. Regarding these two WTG inertial control methods, detailed analysis and comparison are conducted in papers [2], [6] and [7]. Both FBIC and SIC algorithms propose an upper layer controller for the WECS. Therefore, the inertial controller can actively manipulate the active power reference of machine side converter of WTG based on system frequency changes, and the kinetic energy within a WTG can be released as inertial response during frequency events in a controlled manner. As a result, both FBIC and SIC for WTG can be categorized as active inertial control methods.

The method of VSG is also one of the inertial control techniques that integrates virtual inertia into a distributed generator (DG) [8]. As articulated in [8], VSG incorporates the mathematical model of a SG and it can be divided into emulated mechanical part and emulated electrical part. The electrical part of VSG models the excitation system and imitates the output LC filter of inverter system as the stator of a SG. Meanwhile, the mechanical part of VSG implements the swing equation and the governor behavior of a SG into the control of an inverter. Therefore, VSG based DGs are able to participate in the frequency regulation of power systems by providing virtual inertia response. In terms of control behavior, VSG can be viewed as a passive inertial control method in which no extra upper layer controller is required. Referring to the operation of a SG, the power angle of VSG based WTG is passively changed during power system frequency events, so that the kinetic energy within WTG can be released as inertial response. Opposite to the operation of an active inertial control method, VSG does not require frequency error to exceed certain threshold in order to start releasing the inertial response. Hence, the threshold determination process can be eliminated, and inertial control can be enabled in a faster way during system frequency events.

However, the introduction of swing equation using VSG enlarges the time constant of the active power loop (APL) of inverter systems. As a result, with satisfying frequency support, slower dynamics and larger oscillations can be observed which makes original VSG unsuitable for converting fast varying renewable source, wind power for instance, to electric power. In fact, the scheme of adaptive inertial constant and damping is proposed in many papers in order to improve the dynamic response and settling time of a VSG. However, it is difficult to guarantee that the swing equation can switch back to slow dynamics mode to release inertial response 


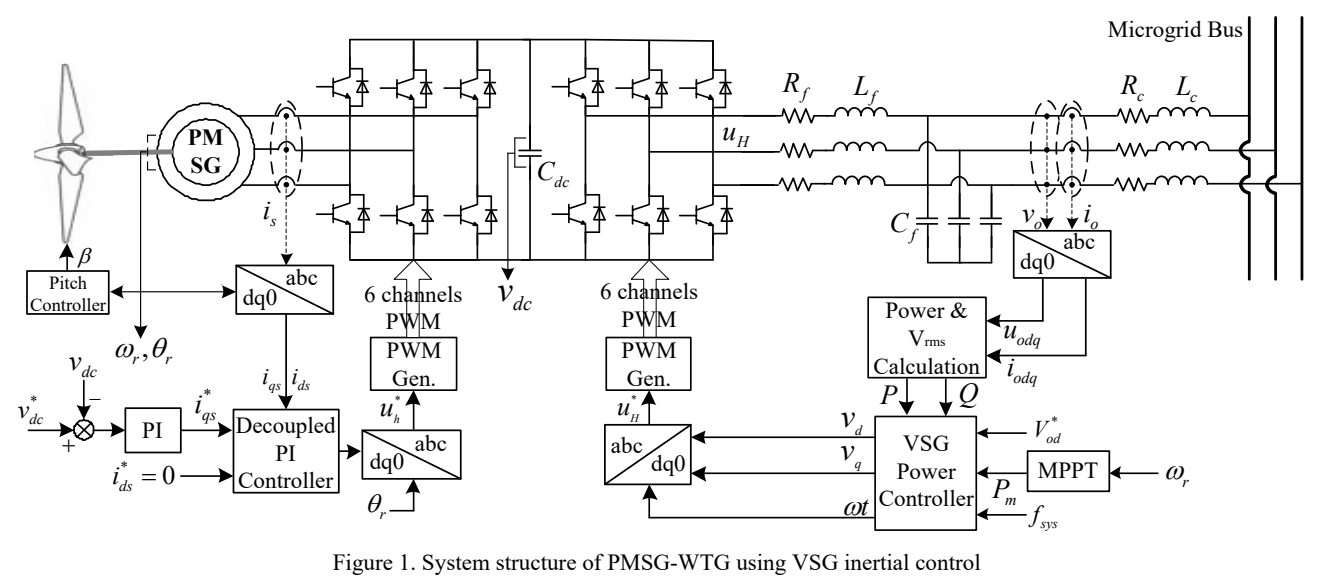

before frequency events due to the uncertainty of power systems. Currently, most applications of implementing VSG algorithm within DGs depend on or benefit from ESS [9]. In paper [10], the operation and inertial response of a PMSG WTG using VSG control is conducted and analyzed. However, the performance of VSG control under variable wind speed condition is not included, which will be further analyzed in this paper. In this paper, the inertial control algorithm using VSG with multiple virtual rotating masses (MVRMs) is proposed for PMSG-WTG. Besides, the nonlinear model and its small-signal linearization of PMSGWTG integrated with VSG are derived. The control performance of the PMSG-WTG is verified in a modified 10MVA IEEE 14 bus system.

\section{MODELING OF VSG BASEd PMSG-WTG}

The VSG based inertial control for PMSG-WTG is presented in Fig.1. $R_{f}, L_{f}, C_{f}$ are resistance, inductance, and capacitance of inverter LC filter and $R_{c}, L_{c}$ are lumped transformer and line impedance. As shown in Fig. 1, PMSG is directly driven by the rotor of wind turbine. Machine side converter (MSC) regulates the voltage of DC link as a rectifier while grid side converter (GSC) operates as a VSG. The active power reference of GSC is calculated through MPPT as emulated mechanical power of VSG. The pitch controller uses PI controller. The model of the VSG controller under $d-q$ reference frame can be expressed in the following equations (1)-(2). Based on equation (2), the VSG is controlled as a PV node as in power flow studies.

$$
\begin{gathered}
\Delta \dot{\omega}=-\frac{\left(P-P_{m}\right)}{2 H_{V S G} \omega_{n}}-\frac{D_{p}\left(\omega-\omega_{g}\right)}{2 H_{V S G}} \\
\dot{v}_{o d}=-\frac{\omega_{n} n}{0.5 v_{d c}}\left(v_{o d}-V_{o d}^{*}\right)
\end{gathered}
$$

Here, $\omega_{g}$ and $\omega$ are grid frequency and VSG operating frequency (emulated generator rotating speed) in radians individually, where $\omega=\Delta \omega+\omega_{g}$. And $\omega_{n}$ is nominal frequency in radians. $H_{V S G}$ is the inertial constant and $D_{p}$ is the damping factor from swing equation of VSG. Besides, $n$ is the integrator gain in VSG's excitation system. $V_{o d}{ }^{*}$ is the nominal voltage and $v_{o d}$ is the voltage feedback of PMSG-WTG, respectively. And $v_{o q}$ can be assigned as 0 for simplicity. Referring to Figure 1, $P$ and $P_{m}$ represent active power output and active power reference, which are emulated electromagnetic power and emulated mechanical power of VSG, respectively. Furthermore, $P_{m}$ is determined based on MPPT with respect to the rotating speed of the rotor of wind turbine $\omega_{r}$, which can be formulated in equation (3).

$$
P_{m}=K \omega_{r}{ }^{3}
$$

where, referring to paper [6] and [11], constant $K$ is:

$$
K=\frac{1}{2} \rho R^{5} \frac{C_{p \max }}{\lambda_{\text {opt }}{ }^{3}}
$$

The active power output $P$ and reactive power output $Q$ of PMSG-WTG can be derived in equation (4) and equation (5). $\omega_{c}$ is the cut-off frequency of low-pass filters.

$$
\begin{aligned}
& \dot{P}=-\omega_{c} P+1.5 \omega_{c} v_{o d} i_{o d} \\
& \dot{Q}=-\omega_{c} Q+1.5 \omega_{c} v_{o d} i_{o q}
\end{aligned}
$$

The dynamics of the output current of PMSG-WTG considering the lumped impedance can be obtained through equation (6) below.

$$
\left\{\begin{array}{l}
L_{c} i_{o d}=v_{o d}-v_{g d}-R_{c} i_{o d}+\omega L_{c} i_{o q} \\
L_{c} i_{o q}=-v_{g q}-R_{c} i_{o q}-\omega L_{c} i_{o d}
\end{array}\right.
$$

At the machine side of PMSG-WTG, the model of rotor speed of wind turbine $\omega_{r}$ can be written as in equation (7).

$$
\dot{\omega}_{r}=\frac{\left(T_{w}-T_{e}\right)}{2 H_{w}}
$$

Here, $H_{w}$ is the combined inertial constant of wind turbine and PMSG. $T_{w}$ represents aerodynamic mechanical torque input and $T_{e}$ is the electromagnetic torque of PMSG. It is worth mentioning that the electromagnetic power of PMSG $P_{e}$, which equals to $\omega_{r} T_{e}$, is different from the electromagnetic torque of VSG $P$. The energy difference between MSC and GSC is absorbed or compensated by capacitor at DC link of PMSG-WTG. In this paper, zero d-axis current (ZDC) control is employed for the regulation of MSC [12]. Therefore, electromagnetic torque $T_{e}$ can be presented in equation (8).

$$
T_{e}=1.5 n_{p} \psi_{f} i_{s q}
$$

As presented, a round pole PMSG is used in this paper. $\psi_{f}$ is the rotor flux linkage of PMSG in RMS value, meanwhile $n_{p}$ is the number of pole pairs. $i_{s q}$ represents the current component along with q-axis of PMSG's stator, which can be expressed in equation (9). Equation (9) is valid when the 
system poles that associated with the inner loop PI controller of MSC have fast dynamics [12].

$$
\dot{i}_{s q}=-k_{m p} \dot{v}_{d c}-k_{m i} v_{d c}+k_{m i} v_{d c}{ }^{*}
$$

Here, $k_{m p}$ and $k_{m i}$ are proportional and integral factors of outer loop PI controller. $v_{d c}{ }^{*}$ denotes the nominal voltage of system DC link while $v_{d c}$ is the DC voltage measurement. Finally, the dynamic behavior of DC link can be described using equation (10), where $C_{d c}$ is the capacitance at DC link.

$$
\dot{v}_{d c}=\frac{\left(\omega_{r} T_{e}-P\right)}{C_{d c} v_{d c}}
$$

Summarizing equation (1) through equation (10), the dynamic nonlinear model of PMSG-WTG using VSG inertial control can be formulated. Moreover, the small-signal model of proposed system can be written as in equation (11) by applying the first order Taylor's expansion. The system state variables are $\Delta x=\left[\Delta \omega_{r}, \Delta v_{d c}, \Delta i_{s q}, \Delta \delta, \Delta \omega, \Delta P, \Delta v_{o d}, \Delta i_{o d}\right.$, $\left.\Delta i_{o q}\right]^{\mathrm{T}}$, and system inputs are recognized as $\Delta u=\left[\Delta v_{d c}{ }^{*}, \Delta V_{o d}{ }^{*}\right]^{\mathrm{T}}$. In matrix $A$ and $B$, the parameters that are written in uppercase with subscript 0 mean the values of corresponding variables derived from their steady state. $\Delta d=\left[\Delta T_{w}, \Delta \omega_{g}, \Delta v_{g d}, \Delta v_{g q}\right]^{\mathrm{T}}$ are disturbances from either wind input or power systems.

$$
\Delta \dot{x}=A \Delta x+B \Delta u+D \Delta d
$$

Even though the design of inertial constant of VSG can be similar to that of a conventional SG, VSG based PMSG-WTG is still going to suffer from severe synchronizing oscillation and poor tracking performance.

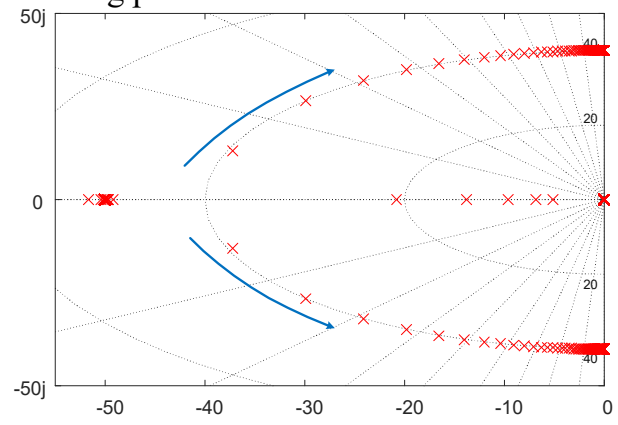

Fig. 2. Trajectory of eigenvalues of VSG controller when $\mathrm{H}_{V S G}$ increases.

Figure 2 shows the trajectory of eigenvalues associated with increasing $H_{V S G}$. The corresponding eigenvalues are approaching the imaginary axis with the enhancement of inertial response. Inevitably, slow dynamics introduced by original VSG causes PMSG-WTG vulnerable to wind variability even though the inertial response of VSG can reach a desirable level, especially in region 2 of the wind turbine. As a result, a larger difference of power flow between MSC and GSC exists and leads to heavier mechanical burden on the rotor shaft of wind turbine.

From the perspective of power regulation of PMSG-WTG, four simulation cases are illustrated in Figure 3, where these four cases are: (a) original VSG control with inertial constant of $H_{V S G}$; (b) original VSG control with inertial constant of $0.5^{*} H_{V S G}$; (c) original VSG control with inertial constant of $0.25^{*} H_{V S G}$; and (d) FBIC. Here, $H_{V S G}=1.56 \mathrm{~s}$. In individual case, the red curve presents the active power output of PMSGWTG and the blue curve shows the active power reference $P_{\text {mppt. }}$ PMSG-WTG is operating in region 2 in all four cases.

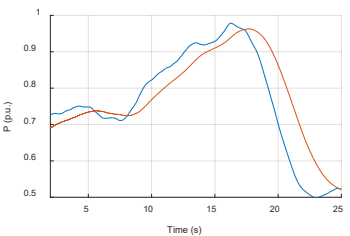

(a)

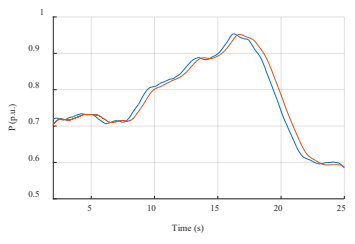

(c)

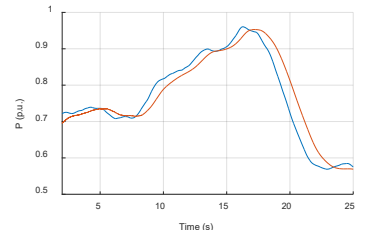

(b)

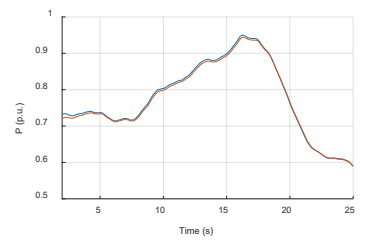

(d)
Fig. 3. Active power output of PMSG-WTG under variable wind speed

In the variable wind speed condition as in Figure 3, the increase of $H_{V S G}$ resulting slower dynamics of VSG which further affects the behavior of the rotor shaft and $P_{\text {mppt }}$ of a WTG. On the other hand, it can be observed from case (a)-(c) that significant improvement can be made by lowering $H_{V S G}$, but it contradicts the initiative of applying a VSG. In PMSG, extra mechanical torque $\Delta T$ can be observed due to imbalance of power flow between MSC and GSC, which can be further demonstrated in later section.

\section{VSG BASED PMSG-WTG WITH MVRMS}

In this section, VSG with MVRMs is proposed so that VSG based PMSG-WTG can respond to fast variation wind speed and still maintain strong inertial capability of a typical VSG system. At the ideal steady state of PMSG-WTG, power balance $P_{w 0}=P_{e 0}=P_{0}$ can be achieved. However, as described in section 2, the original VSG is not capable of smoothly

$\left.\begin{array}{cccc}0 & 0 & 0 & 0 \\ \frac{-1}{C_{d c} V_{d c 0}} & 0 & 0 & 0 \\ \frac{k_{m p}}{C_{d c} V_{d c 0}} & 0 & 0 & 0 \\ 0 & 0 & 0 & 0 \\ \frac{-1}{2 H_{V S G}} & 0 & 0 & 0 \\ -\omega_{c} & 1.5 \omega_{c} I_{o d 0} & 1.5 \omega_{c} V_{o d 0} & 0 \\ 0 & \frac{-\omega_{n} n}{0.5 V_{d c 0}} & 0 & 0 \\ 0 & \frac{1}{L_{c}} & \frac{-R_{c}}{L_{c}} & \omega_{0} \\ 0 & 0 & -\omega_{0} & \frac{-R_{c}}{L_{c}}\end{array}\right], B=\left[\begin{array}{cc}0 & 0 \\ 0 & 0 \\ k_{m i} & 0 \\ 0 & 0 \\ 0 & 0 \\ 0 & 0 \\ 0 & \frac{\omega_{n} n}{0.5 V_{d c 0}} \\ 0 & 0 \\ 0 & 0\end{array}\right], D=\left[\begin{array}{cc}0 & 0 \\ 0 & 0 \\ 0 & 0 \\ 0 & 0 \\ 0 & 0 \\ 0 & 0 \\ 0 & 0 \\ \frac{-1}{L_{c}} & 0 \\ 0 & \frac{-1}{L_{c}}\end{array}\right]$


converting $P_{e}$ from PMSG to inverter output $P$ due to the existing large inertial constant. The change of $P_{w}$ that cannot be instantly consumed by VSG can cause large imbalance of power flow between MSC and GSC. As a result, such imbalance will lead to poor tracking of $P_{m p p t}$, large power output oscillation as well as PMSG-WTG system instability. Furthermore, the increase of force bearing on rotor shaft of wind turbine can be observed, which also can be explained in equation (8) and equation (11). Therefore, it is crucial to introduce a direct path with fast system dynamics to export the variation of power input that cannot be consumed by VSG for PMSG-WTG operating under variable wind speed condition.

Figure 4 illustrates the control diagram of the proposed VSG with MVRMs. In proposed system structure, the power output from PMSG is equivalently sent into three virtual rotating masses (VRMs), where VRM 1 only processes the aerodynamic power input and its fast variation that cannot be instantly processed by GSC. VRM 2 maintains the characteristics of an original VSG meanwhile still processes the majority amount of power. And VRM 3 is a conditional path to boost inertial response when more kinetic energy is available to be released.

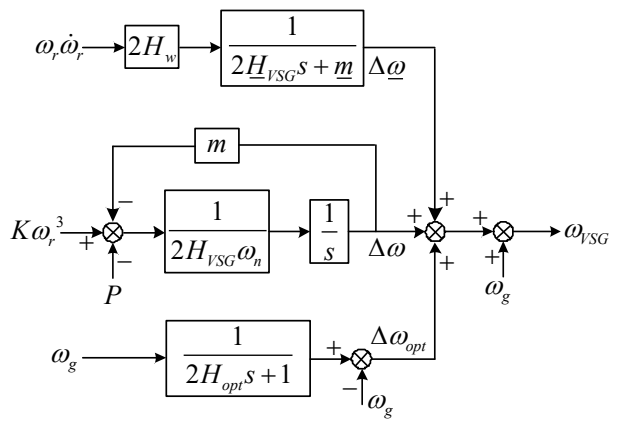

Fig. 4. The control diagram of VSG with MVRMs

In fact, the aerodynamic power $P_{w}$ usually is not available for measurement. Therefore, referring equation (7), the variation of aerodynamic power input that cannot be instantly consumed by VSG is further derived as $P_{w^{-}} P_{e}$ in equation (12)

$$
P_{w}-P_{e}=2 H_{w} \omega_{r} \dot{\omega}_{r}
$$

So, the transfer function of VRM 1 can be expressed in equation (13) by considering swing equation, where $\underline{H}_{V S G}$ and $\underline{m}$ represent the inertial constant and damping factor of VRM 1 , respectively. In order to obtain the fast dynamics such that VRM 1 can follow $P_{w}-P_{e}$, the inertial constant $\underline{H}_{V S G}$ should be small enough. Alternatively, the small value of $\underline{H}_{V S G}$ can be interpreted as the time constant of a low pass filter so that such filter can wash out the noise introduced from the derivative term of equation (12).

$$
\Delta \underline{\omega}=\frac{1}{2 \underline{H}_{V S G} s+\underline{m}}\left(P_{w}-P_{e}\right)
$$

VRM 3 can be considered as an optional path to boost the inertial response of a VSG when more kinetic energy in rotor shaft of PMSG-WTG is available to be extracted. The inertial response can be introduced by VSG because the frequency decreasing rate of a VSG is always relatively lower than the frequency deceasing rate of a power system during frequency dip. Applying the same concept and employing a delay, the dynamic model of VRM 3 can be derived as in equation (14).

$$
\Delta \omega_{o p t}=\omega_{g} e^{-\tau s}-\omega_{g}=\frac{1}{2 H_{o p t} s+1} \omega_{g}-\omega_{g}
$$

According to equation (14), a small delay is introduced so that extra frequency difference between VSG and physical network can be generated during system frequency events. Generally, equation (14) is able to be rewritten which also unifies with the format of the swing equation of a VSG. Thus, inertial constant of VRM 3, $H_{o p t}$, can be calculated based on the desired delay. In this paper, the enabling of VRM 3 is determined by: first, the rotor speed must be bigger than 0.4 p.u; and second, inertial response from VRM 2 is already enabled. It is worth mentioning that the proposed VSG still maintains its passive characteristics. The enabling strategy of VRM 3 only depend on the status of VRM 2. By including VRM 3, the overall swing equation of the proposed VSG can be written as equation (15). The stability of proposed system can be easily proven which is omitted in this paper.

$$
\begin{aligned}
\Delta \dot{\omega}_{V S G}= & -\frac{1}{2 H_{V S G} \omega_{n}}\left(P-K \omega_{r}{ }^{3}\right)-\frac{m}{2 H_{V S G}} \Delta \omega \\
& -\frac{1}{2 \underline{H}_{V S G}}\left(P_{e}-P_{w}\right)-\frac{\underline{m}}{2 \underline{H}_{V S G}} \Delta \underline{\omega} \\
& -\Delta \omega_{g}-\frac{1}{2 H_{o p t}} \Delta \omega_{o p t}
\end{aligned}
$$

\section{INERTIAL CONTROLS SIMULATIONS AND EVALUATIONS}

In this section, the inertial control methods are simulated and evaluated in a modified IEEE 14 bus testbed. Different from a typical IEEE 14 bus testbed, the voltage of the modified testbed is $10 \mathrm{kV}$ in order to accommodate the application of a microgrid. The line impedances are also scaled down and the overall load is $7.015 \mathrm{MW}$ and $1.82 \mathrm{MVar}$. A $0.7 \mathrm{MW}$ and $0.1 \mathrm{MVar}$ load disturbance is introduced at bus 4 and proposed VSG based 2MVA PMSG-WTG [11] is placed at bus 14 .

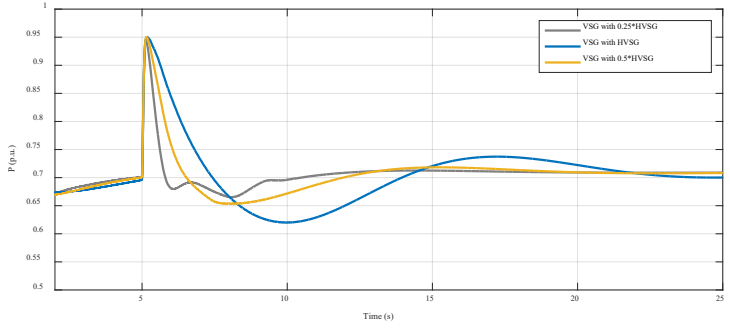

Fig. 5. Active power output of PMSG-WTG during frequency event

Figure 5 demonstrates the inertial response of PMSGWTG using conventional VSG when different inertial constants are used $\left(H_{V S G}=1.56 \mathrm{~s}\right)$. At $5 \mathrm{~s}$, the disturbance load is connected to the grid. As we can see, the inertial response achieved by VSG can react instantly with very high active power ramping rate. Generally, VSG inertial control can have faster response than FBIC [2], [6], [7]. Hence, VSG can be considered as an effective inertial control method with fast inertial response. However, major issues can be encountered for the application of VSG under variable wind speed condition. First, wind profile in Figure 6 is used in this paper, whose mean value is $10 \mathrm{~m} / \mathrm{s}$. 


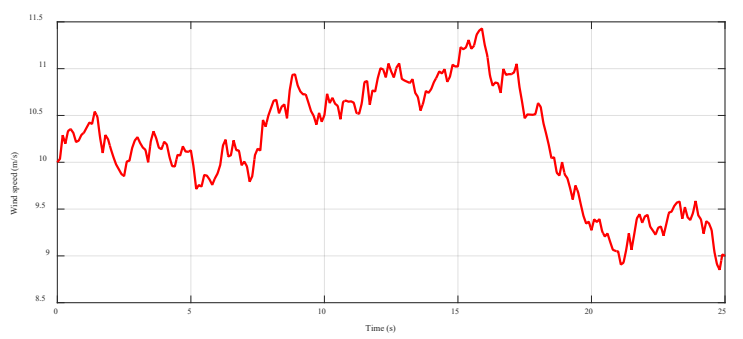

Fig. 6. Variable wind speed data

Extra torque bearing on the rotor shaft of PMSG-WTG due to the employment of VSG is shown in Figure 7.

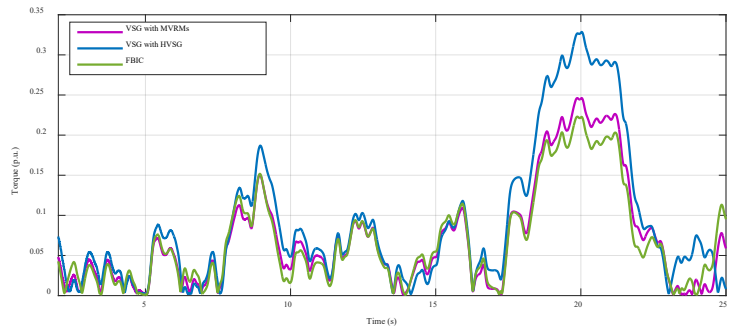

Fig. 7. $\Delta T$ applied on the rotor shaft of PMSG-WTG

During the normal operation of a WTG, FBIC is disenabled and traditional controls on MSC and GSC are still used. Thus, the green curve (FBIC) can be considered as the base torque when no frequency event is triggered. The results in Figure 7 further proves the conclusion in section 2 that more bearing force on the rotor shaft of PMSG-WTG can be observed by directly using VSG. On the other hand, comparing the case using original VSG (blue), it is obvious that the rotor shaft of PMSG-WTG can experience less torque due to the employment of MVRMs (purple). The torque bearing level of rotor shaft using VSG with MVRMs is close to that of PMSG-WTG using FBIC. Furthermore, at the time instant of $7 \mathrm{~s}$ and $24 \mathrm{~s}$, PMSG-WTG can even experience less torque comparing with PMSG-WTG using FBIC.
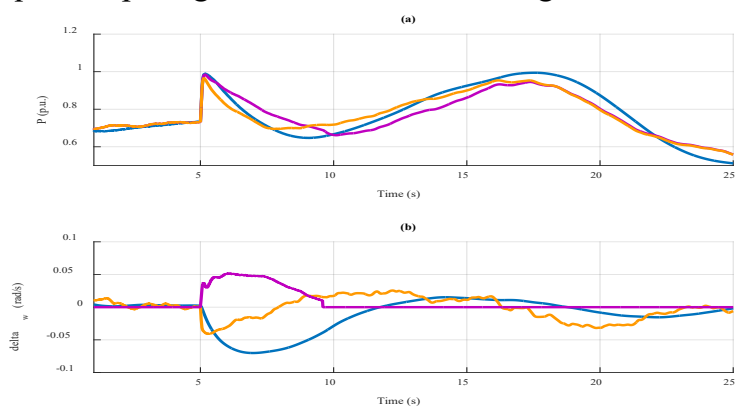

Fig. 8. Reponses of inertial controls under variable wind speed

Figure 8 depicts the dynamic behaviors of individual VRM of proposed VSG control. In part (a), the blue curve represents the operation of original VSG. Meanwhile, the orange curve shows the operation of proposed VSG when only VRM 1 and 2 are functioning. The purple curve shows the operation of proposed VSG when all VRMs are operating. In part (b), the rotating speed difference of VRM 1 (orange), VRM 2 (blue), and VRM 3 (purple) comparing with grid frequency are shown. According to Figure 8, the degradation of inertial response by introducing VRM 1 is negligible whereas the performance of active power tracking can be vastly improved. VRM 1 also benefits the damping of power oscillations in VSG such that smoother recovery process can be obtained. On the other hand, the start-up of VRM 3 can further boost the inertial control, which is shown in the (b) part of Figure 8. Overall, a better inertial control performance can be achieved by the proposed VSG with MVRMs.

\section{CONCLUSION}

PMSG-WTG system using VSG based inertial control is modeled and analyzed. The overall system nonlinear dynamic model and its small signal linearization model are derived. In this paper, aiming at the problem that existing VSG with desirable inertial response cannot be adaptive to fast changing wind condition, VSG with MVRMs is proposed in order to improve the active power tracking performance as well as to boost inertial control of a VSG. Proposed method is verified in modified 10MVA IEEE 14 bus system. Simulation results show that the participation of MVRMs can greatly improve the active power tracking performance of a VSG while desirable inertial response is still maintained.

\section{REFERENCES}

[1] J. Lee, E. Muljadi, P. Srensen and Y. C. Kang, "Releasable Kinetic Energy-Based Inertial Control of a DFIG Wind Power Plant," in IEEE Transactions on Sustainable Energy, vol. 7, no. 1, pp. 279-288, Jan. 2016.

[2] Wang, X., Gao, W., Wang, J., et al.: "Inertial response of wind power plants: a comparison of frequency-based inertial control and stepwise inertial control". North American Power Symp. (NAPS), Denver, Colorado, 2016, pp. 1-6.

[3] Z. Wu, D. W. Gao, H. Zhang, S. Yan and X. Wang, "Coordinated Control Strategy of Battery Energy Storage System and PMSG-WTG to Enhance System Frequency Regulation Capability," IEEE Transactions on Sustainable Energy, vol. 8, no. 3, pp. 1330-1343, July 2017.

[4] Kang, M., Muljadi, E., Hur, K., et al.: 'Stable adaptive inertial control of a doubly-fed induction generator', IEEE Trans. Smart Grid, 2016, 7 , (6), pp. 2971-2979.

[5] Kang, M., Kim, K., Muljadi, E., et al.: 'Frequency control support of a doubly-fed induction generator based on the torque limit', IEEE Trans. Power Syst., 2016, 31, (6), pp. 4575-4583.

[6] X. Wang, W. Gao, A. Scholbrock, E. Muljadi, V. Gevorgian, J. Wang, W. Yan and H. Zhang, "Evaluation of different inertial control methods for variable-speed wind turbines simulated by fatigue, aerodynamic, structures and turbulence (FAST)," IET Renewable Power Generation, vol. 11, no. 12, pp. 1534-1544, 2017.

[7] X. Wang, W. Gao, W. Yan, J. Wang, E. Muljadi, V. Gevorgian, and A. Scholbrock. "Evaluation of the inertial response of variable-speed wind turbines using advanced simulation," in Proc. 2017 IEEE Power \& Energy Society General Meeting, Chicago, IL, 2017, pp. 1-5.

[8] Q. C. Zhong and G. Weiss, "Synchronverters: Inverters That Mimic Synchronous Generators," IEEE Trans. on Industrial Electronics, vol. 58, pp. 1259-1267, Apr. 2011.

[9] X. Wang, M. Yue and E. Muljadi, "PV generation enhancement with a virtual inertia emulator to provide inertial response to the grid," 2014 IEEE Energy Conversion Congress and Exposition (ECCE), Pittsburgh, PA, 2014, pp. 17-23.

[10] J. Xi, H. Geng, G. Yang, and S. Ma, "Inertial response analysis of PMSG-based WECS with VSG control" The Journal of Engineering, vol. 2017, no.13, pp. 897-901, Oct. 2017.

[11] B. Wu, Y. Lang, N. Zargari, and S. Kouro, Power Conversion and Control of Wind Energy Systems. Wiley-IEEE Press, 2011.

[12] N. Pogaku, M. Prodanovic and T. C. Green, "Modeling, analysis and testing of autonomous operation of an inverter-based microgrid," IEEE Trans. on Power Electronics, vol. 22, pp. 613-625, Mar. 2007. 